\title{
Factor XII Inactivation
}

National Cancer Institute

\section{Source}

National Cancer Institute. Factor XII Inactivation. NCI Thesaurus. Code C131669.

Presence of inactivating antibodies to factor XII in the blood. 